\title{
Short-range correlations in low-lying nuclear excited states
}

\author{
S.R. Mokhtar ${ }^{1,3}$, G. Co ${ }^{1,2}$ and A. M. Lallena ${ }^{4}$ \\ 1) Dipartimento di Fisica, Università di Lecce, I-73100 Lecce, Italy \\ 2) Istituto Nazionale di Fisica Nucleare sez. di Lecce, I-73100 Lecce, Italy \\ 3) Department of Physics, University of Assiut, Assiut, Egypt \\ ${ }^{4)}$ Departamento de Física Moderna, Universidad de Granada, E-18071 Granada, Spain
}

\begin{abstract}
The electromagnetic transitions to various low-lying excited states of ${ }^{16} \mathrm{O},{ }^{48} \mathrm{Ca}$ and ${ }^{208} \mathrm{~Pb}$ are calculated within a model which considers the short-range correlations. In general the effects of the correlations are small and do not explain the required quenching to describe the data.
\end{abstract}

PACS number(s): 21.60.-n, 25.30.Dh

The description of the electron scattering form factors of low-lying excited states is still an unsolved problem. The disagreement between theory and experiment is particularly annoying for the high angular momentum stretched states which are composed by few particle-hole (p-h) configurations. Because of this relatively simple structure one expects that the independent particle model (IPM) should be able to reproduce the data. This is not far to be true for what concerns the shape of the form factors, but the theoretical results usually overestimate the data. The disagreement between theory and experiment is commonly summarized by a single number: the quenching factor $Q$, necessary to reproduce the data.

There have been various attempts to identify the sources of this disagreement, but the situation has not yet been clarified. The quenching produced by first order core polarization mechanism has been proposed in Ref. 1] but other studies [2] found this effect to be small. The quenchings of the $12^{-}$and $14^{-}$form factors in ${ }^{208} \mathrm{~Pb}$ have been explained by a Random Phase Approximation (RPA) plus particle vibration coupling model in Ref. [3] but these results have not been confirmed by a self consistent first- and second- RPA calculation 何.

In Ref. [5] a mechanism related to the presence of short-range correlations has been proposed. These correlations modify the occupation probability of the single particle levels, reducing the occupation of the hole states and producing a finite probability of occupying the particle states even when the nucleus is in its ground state. This idea is supported by the fact that elastic electron scattering data [6] and (e,e'p) data [7] in ${ }^{208} \mathrm{~Pb}$ can be explained assuming partial occupation probability of the single particle levels closed the Fermi surface [8].

In this work we have studied the electron excitation of some non collective low-lying states of doubly closed shell nuclei with a model that takes into account the short-range correlations. The starting point of our model is the CBF ansatz for the description of the nuclear ground state:

$$
\left|\Psi_{0}\right\rangle=G\left|\Phi_{0}\right\rangle
$$

where $G$ is a correlation function and $|\Phi\rangle$ is a Slater determinant of single particle wave functions. The many-body responses induced by an operator $O(\mathbf{q})$ can be written as:

$$
\begin{aligned}
S(\mathbf{q}, \omega) & =-\frac{I m}{\pi} \frac{\left\langle\Psi_{0}\left|O^{+}(\mathbf{q})\left(H-E_{0}-\omega+i \eta\right)^{-1} O(\mathbf{q})\right| \Psi_{0}\right\rangle}{\left\langle\Psi_{0} \mid \Psi_{0}\right\rangle} \\
& =-\frac{I m}{\pi} \sum_{n} \xi_{n}^{+}(\mathbf{q})\left(E_{n}-E_{0}-\omega+i \eta\right)^{-1} \xi_{n}(\mathbf{q}),
\end{aligned}
$$

where:

$$
\xi_{n}(\mathbf{q})=\frac{\left\langle\Psi_{n}|O(\mathbf{q})| \Psi_{0}\right\rangle}{\left\langle\Psi_{n} \mid \Psi_{n}\right\rangle^{\frac{1}{2}}\left\langle\Psi_{0} \mid \Psi_{0}\right\rangle^{\frac{1}{2}}}
$$

In the above equations $H$ indicates the hamiltonian, $\mathbf{q}$ and $\omega$ the momentum and energy transfer respectively and $E$ the energies of the nuclear system.

In our model the excited states are defined in analogy with Eq. (1): $\left|\Psi_{n}\right\rangle=G\left|\Phi_{n}\right\rangle$. The correlation $G$ is the same used to describe the ground state. With this ansatz we rewrite Eq. (3) as:

$$
\xi_{n}(\mathbf{q})=\frac{\left\langle\Phi_{n}\left|G^{+} O(\mathbf{q}) G\right| \Phi_{0}\right\rangle}{\left\langle\Phi_{0}\left|G^{+} G\right| \Phi_{0}\right\rangle}\left[\frac{\left\langle\Phi_{0}\left|G^{+} G\right| \Phi_{0}\right\rangle}{\left\langle\Phi_{n}\left|G^{+} G\right| \Phi_{n}\right\rangle}\right]^{\frac{1}{2}} .
$$


In principle the correlation function $G$ has a complicated operatorial structure, analogous to that of the nuclear hamiltonian. In the present work we have considered a purely scalar correlation function which is therefore commuting with the excitation operator $O(\mathbf{q})$. The functional dependence of the correlation function is the Jastrow ansatz [9]:

$$
G(1,2 \ldots A)=\prod_{i<j} f\left(r_{i j}\right),
$$

where $f$ is a two-body correlation function and $r_{i j}$ is the distance between two nucleons. We introduce a function $h_{i j}=f_{i j}^{2}-1$ and we perform a cluster expansion [10] retaining only those terms where the function $h_{i j}$ appears only once. We have tested the model by comparing its results with those of the full calculations. A first comparison has been done for the nuclear matter quasi-elastic charge responses [11] and has shown the validity of our approximation. The model has also been used to evaluate the ground state charge density distributions 12, 13. of various doubly closed shell nuclei. Even in this case the agreement with the results of the full calculations is very good.

In the present work we apply our model to describe the excitation of low-lying states induced by electron scattering. A detailed description of the model can be found in Ref. [14] where the explicit expressions of the transition matrix elements are given. We should remark however that while in the calculations of Ref. [14] the convection current was considered only at the mean-field level, in the present calculations, we have also evaluated all the correlated terms related to this current.

Our model requires two inputs, the set of single particle wave functions and the correlation function. They are not independent since, for a given hamiltonian, they are fixed through the variational principle by minimizing the energy expectation value. The single particle wave functions and the correlations we have used have been taken from Ref. [15] where energy minima of several doubly magic nuclei for the semi-realistic S3 interaction of Afnan and Tang have been found.

With this input we have calculated the electron excitations of various low-lying states in ${ }^{16} \mathrm{O},{ }^{48} \mathrm{Ca}$ and ${ }^{208} \mathrm{~Pb}$ nuclei and we have compared our results with the experimental data of Refs. [16]- [19].

For magnetic excitations the comparison is done with the transverse form factor which can be unambiguously obtained for each cross section value. In the electric excitations, both longitudinal and transverse form factors contribute. Their extraction from the cross section data requires at least two measurements done at same value of the momentum transfer q. Because of the difficulties related to this procedure [20], for the electric states we preferred to compare our calculations directly to the cross sections.

Since our model cannot describe collective effects, we have considered only those states characterized by one or at most two p-h excitations. We have estimated the degree of collectivity of the various excited states by making discrete RPA calculations with both a density dependent Landau-Migdal interaction [21] and the Jülich-Stony Brook interaction [22]. We have selected those states having at least one p-h transition with $X$ amplitude value larger than 0.9 in both calculations.

From this analysis we found three states dominated by two p-h pairs. For these cases we have supposed that the wave function of the excited uncorrelated state could be described as a linear combination of the two p-h pairs:

$$
\left|\Phi_{n}\right\rangle=X_{p h}\left|\Phi_{p h}\right\rangle+X_{p^{\prime} h^{\prime}}\left|\Phi_{p^{\prime} h^{\prime}}\right\rangle
$$

where the $X_{p h}$ amplitudes have been taken from the RPA solution and the other one have been fixed such that $X_{p h}^{2}+X_{p^{\prime} h^{\prime}}^{2}=1$.

The results of our calculations are summarized in Table 1. The quenching factors $Q$ for the uncorrelated (IPM) and correlated calculations and the $\chi^{2}$ per datum are compared. The values of the $\chi^{2}$ have been evaluated after the application of the quenching factors to the original results.

The short-range correlations do not substantially change the IPM results. In some cases, the correlations reduce the cross section values, therefore the quenching factors increase. There are, however, various situations where the correlations effects go in the opposite direction.

We show in Figs. 1 and 2 the results obtained for the $4^{-}$state in ${ }^{16} \mathrm{O}$ and for the $12^{+}$in ${ }^{208} \mathrm{~Pb}$ to make more explicit some of the features of the results. The linear scale used in some panel of the two figures allows for a better identification of the correlations effects consisting in a more or less pronounced modification of the maximum of the distribution. The results are also plotted in a logarithmic scale since they are commonly presented in this way. In the $4^{-}$case the correlations enhance the value of the maximum. This implies a smaller $Q$ for the correlated calculation, as it is shown in the table. Even after the $Q$ factor has been applied the agreement with the data is rather poor, as the high values of the $\chi^{2}$ indicate.

In Fig. 2 the $12^{+}$cross sections calculated for two different values of the scattering angle are presented and compared with the data of Ref. [18]. In this case the correlation lowers the maximum of the distribution. The $12^{+}$excitation has been calculated as a pure neutron $1 \mathrm{i}_{11 / 2} 1 \mathrm{i}_{13 / 2}^{-1}$ transition. The difference in the lowering produced by the correlation 
in panels $a$ and $b$ of the figure, i.e. the ratio between full and dashed lines, is within the $1 \%$. This small difference is produced by the presence of the longitudinal response generated by the electric neutron form factor of Ref. [23] which we use in our calculations.

One can notice that the quenching factors required to reproduce the data are different for the two different scattering angles. However these two values are statistically compatible once the experimental uncertainties are considered.

From what we have presented it appears evident that the correlation effects are very small and cannot be considered relevant for the description of the experimental data. The size of these effects is in agreement with the results of microscopic nuclear matter calculations done within the Correlated Basis Function theory and using the Fermi Hypernetted Chain resummation techniques [24] which find occupation numbers very close to those of the IPM.

It is possible that some of the correlation components we have neglected, especially the tensor terms, could produce noticeable effects, as indicated in Ref. [25]. On the other hand, microscopic calculations in both infinite systems [26] and finite nuclei [27], show that the scalar term is by far the largest one in the correlation. Furthermore, a study of the ground state charge and momentum distributions of doubly closed shell nuclei [13] indicate the small influence of these state dependent terms.

Our experience in RPA calculations [28] has shown that the presence of small amplitude p-h pairs can heavily modify the size, and sometime even the shape, of the form factor. For this reason we think that the origin of the quenching factor should be searched by looking with more detail at the coupling of the single particle excitations with the collective modes of the nucleus.

This work has been partially supported by the CICYT-INFN agreement and by the DGES (PB98-1367) and the Junta de Andalucía (FQM225).

[1] I. Hamamoto, J. Lichtenstadt and G. Bertsch, Phys. Lett. 93B, 213 (1980); ibid. 96B, 249 (1980).

[2] T. Suzuki and H. Hyuga, Nucl. Phys. A402, 491 (1983).

[3] S. Krewald and J. Speth, Phys. Rev. Lett. 45, 417 (1980).

[4] J. Dechargé, L. Sips and D. Gogny, Phys. Lett. 98B, 229 (1981).

[5] V.R. Pandharipande, C.N. Papanicolas and J. Wambach, Phys. Rev. Lett. 53, 1133 (1984).

[6] J.M. Cavedon et al., Phys. Rev. Lett. 49, 978 (1982).

[7] E.N.M. Quint, Ph.D. thesis, University of Amsterdam, 1988 (unpublished); I. Bobeldijk et al., Phys. Rev. Lett. 73, 2684 (1994).

[8] C. Papanicolas in Nuclear Structure at high spin, excitation and momentum transfer, H. Nann ed., (American Institute of Physics, New York 1986).

[9] R. Jastrow, Phys. Rev. 98, 1479 (1955).

[10] S. Fantoni and V.R. Pandharipande, Nucl. Phys. A473, 234 (1987).

[11] J.E. Amaro, A.M. Lallena, G. Co' and A. Fabrocini, Phys. Rev. C 57, 3473 (1998).

[12] G. Co', Nuovo Cimento A108, 623 (1995).

[13] F. Arias de Saavedra, G. Co' and M. M. Renis, Phys. Rev. C 55, 673 (1997).

[14] G. Co' and A. M. Lallena, submitted to Ann. of Phys. (NY).

[15] F. Arias de Saavedra, G. Co', A. Fabrocini and S. Fantoni, Nucl. Phys. A605, 359 (1996).

[16] C.E. Hyde-Wright et al., Phys. Rev. C 35, 880 (1987); C.E. Hyde-Wright, Ph.D. Thesis, MIT 1984 (unpublished).

[17] J.E. Wise et al., Phys. Rev. C 31, 1699 (1985).

[18] J. Lichtenstadt et al., Phys. Rev. Lett. 40, 1127 (1978); J. Lichtenstadt et al., Phys. Rev. C 20, 497 (1979); J. Lichtenstadt, Ph.D. thesis, MIT 1979 (unpublished).

[19] J.P. Connelly et al., Phys. Rev. C 45, 2711 (1992).

[20] G. Co' and J. Heisenberg, Phys. Lett. 197B, 489 (1987).

[21] G. Rinker and J. Speth, Nucl. Phys. A306, 360 (1978).

[22] J. Speth, V. Klemt. J. Wambach and G.E. Brown, Nucl. Phys. A 343, 382 (1980).

[23] G. Hoeler et al., Nucl. Phys. B 114, 505 (1976).

[24] O. Benhar, A. Fabrocini and S. Fantoni, Phys. Rev. C 41, R24 (1990).

[25] A. Fabrocini, Phys. Rev. C 55, 338 (1997).

[26] R. B. Wiringa, V. Ficks and A. Fabrocini, Phys. Rev. C 38, 1010 (1988); A. Akmal, V. R. Pandharipande and D. G. Ravenhall, Phys. Rev. C 58, 1804 (1998).

[27] A. Fabrocini, F. Arias de Saavedra and G. Co', Phys. Rev. C 61, 044302 (2000)

[28] A.M. Lallena, Nucl. Phys. A489, 70 (1988); G. Co' and A.M. Lallena, ibid. A510, 139 (1990); N.M. Hintz, A.M. Lallena and A. Sethi, Phys. Rev. C 45, 1098 (1992); F. Arias de Saavedra and A.M. Lallena, Phys. Rev. C 50, 2384 (1994). 
TABLE I. Excited states calculated and their quenching factors. We have indicated the excitation energy $E$, the p-h pairs considered, the $X$ amplitudes, Eq. (6), the quenching factors $Q$ and the $\chi^{2}$ for both calculations, and the reference where the experimental points have been taken. For the electric states we have considered the quenching on the full cross section, and we have indicated the scattering angle were the cross section has been measured.

\begin{tabular}{|c|c|c|c|c|c|c|c|c|c|}
\hline & \multirow[t]{2}{*}{$\mathrm{J}^{\pi}$} & \multirow[t]{2}{*}{$E(\mathrm{MeV})$} & \multirow[t]{2}{*}{ p-h pairs } & \multirow[t]{2}{*}{$X_{p h}$} & \multicolumn{2}{|c|}{ IPM } & \multicolumn{2}{|c|}{ Correlated } & \multirow[t]{2}{*}{ Ref. } \\
\hline & & & & & $Q$ & $\chi^{2}$ & $Q$ & $\chi^{2}$ & \\
\hline${ }^{16} \mathrm{O}$ & $4^{-}$ & 18.98 & $\begin{array}{ll}\pi 1 \mathrm{~d}_{5 / 2} & 1 \mathrm{p}_{3 / 2}^{-1} \\
\nu 1 \mathrm{~d}_{5 / 2} & 1 \mathrm{p}_{3 / 2}^{-1}\end{array}$ & $\begin{array}{l}+0.7058 \\
-0.7084\end{array}$ & 0.67 & 26.35 & 0.66 & 35.06 & 16 \\
\hline${ }^{48} \mathrm{Ca}$ & $4^{-}$ & 6.105 & $\begin{array}{ll}\pi 1 \mathrm{f}_{7 / 2} & 2 \mathrm{~s}_{1 / 2}^{-1} \\
\pi 1 \mathrm{f}_{7 / 2} & 1 \mathrm{~d}_{3 / 2}^{-1}\end{array}$ & $\begin{array}{l}-0.7520 \\
-0.6591\end{array}$ & 0.61 & 16.95 & 0.61 & 16.98 & 17 \\
\hline & $6^{-}$ & 8.557 & $\pi 1 \mathrm{f}_{7 / 2} 1 \mathrm{~d}_{5 / 2}^{-1}$ & 1.0 & 0.06 & 6.99 & 0.06 & 8.67 & 17 \\
\hline & $3^{+}$ & 4.608 & $\nu 2 \mathrm{p}_{3 / 2} 1 \mathrm{f}_{7 / 2}^{-1}$ & 1.0 & 0.32 & 1.93 & 0.34 & 2.26 & 17 \\
\hline & $5^{+}$ & 5.147 & $\nu 2 \mathrm{p}_{3 / 2} 1 \mathrm{f}_{7 / 2}^{-1}$ & 1.0 & 0.42 & 20.77 & 0.43 & 19.83 & 17 \\
\hline$\theta=160^{\circ}$ & $5^{-}$ & 8.804 & $\begin{array}{lll}\nu & \lg _{9 / 2} & 1 \mathrm{f}_{7 / 2}^{-1} \\
\pi & 1 \mathrm{f}_{7 / 2} & 1 \mathrm{~d}_{3 / 2}^{-1}\end{array}$ & $\begin{array}{l}-0.8242 \\
+0.5663\end{array}$ & 0.34 & 7.11 & 0.37 & 8.01 & 17 \\
\hline${ }^{208} \mathrm{~Pb}$ & $9^{+}$ & 5.01 & $\nu 2 \mathrm{~g}_{9 / 2} 1 \mathrm{i}_{13 / 2}^{-1}$ & 1.0 & 0.38 & 5.92 & 0.37 & 7.17 & 19 \\
\hline & $12^{-}$ & 7.064 & $\pi 1 \mathrm{i}_{13 / 2} 1 \mathrm{~h}_{11 / 2}^{-1}$ & 1.0 & 0.28 & 7.64 & 0.27 & 8.85 & 19 \\
\hline & $14^{-}$ & 6.745 & $\nu 1 \mathrm{j}_{15 / 2} 1 \mathrm{i}_{13 / 2}^{-1}$ & 1.0 & 0.39 & 5.53 & 0.39 & 5.53 & 19 \\
\hline$\theta=90^{\circ}$ & $10^{+}$ & 5.920 & $\nu 1 \mathrm{i}_{11 / 2} 1 \mathrm{i}_{13 / 2}^{-1}$ & 1.0 & 0.63 & 18.90 & 0.69 & 20.63 & 18 \\
\hline$\theta=160^{\circ}$ & $10^{+}$ & 5.920 & $\nu 1 \mathrm{i}_{11 / 2} 1 \mathrm{i}_{13 / 2}^{-1}$ & 1.0 & 0.88 & 28.91 & 0.95 & 32.15 & 18 \\
\hline$\theta=90^{\circ}$ & $12^{+}$ & 6.100 & $\nu 1 \mathrm{i}_{11 / 2} 1 \mathrm{i}_{13 / 2}^{-1}$ & 1.0 & 0.52 & 7.55 & 0.57 & 8.76 & 18 \\
\hline$\theta=160^{\circ}$ & $12^{+}$ & 6.100 & $\nu 1 \mathrm{i}_{11 / 2} 1 \mathrm{i}_{13 / 2}^{-1}$ & 1.0 & 0.39 & 12.70 & 0.42 & 14.84 & 18 \\
\hline
\end{tabular}



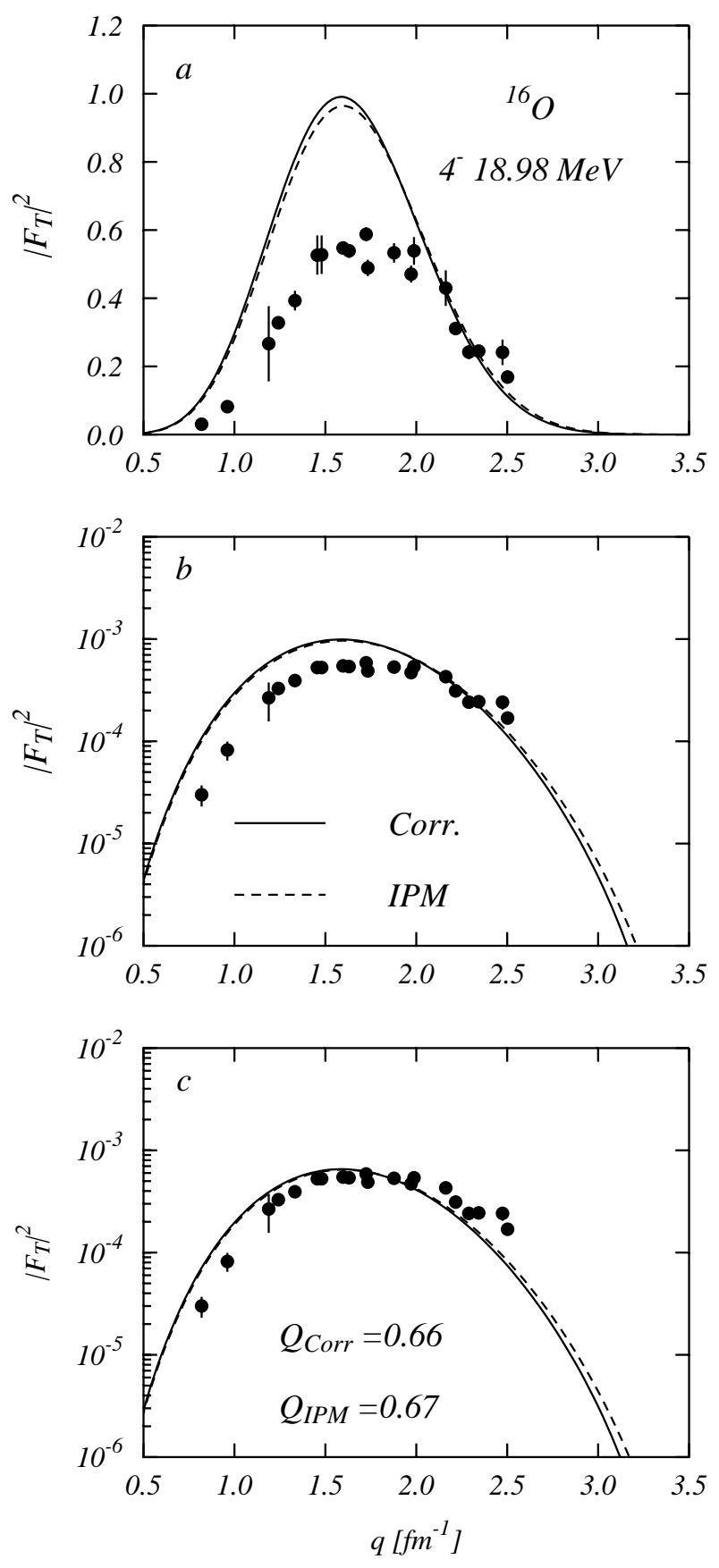

FIG. 1. Transverse form factor for the $4^{-}$in ${ }^{16} \mathrm{O}$ state at $18.98 \mathrm{MeV}$. In the panel $a$ the results of the IPM (dashed line) and of our correlated model (full line) are compared with the data of Ref. 16]. The same figure is redrawn in panel $b$ in log scale since these results are commonly presented in this way. The panel $c$ show the comparison between theoretical results and experimental data, after the quenching factors have been applied. 

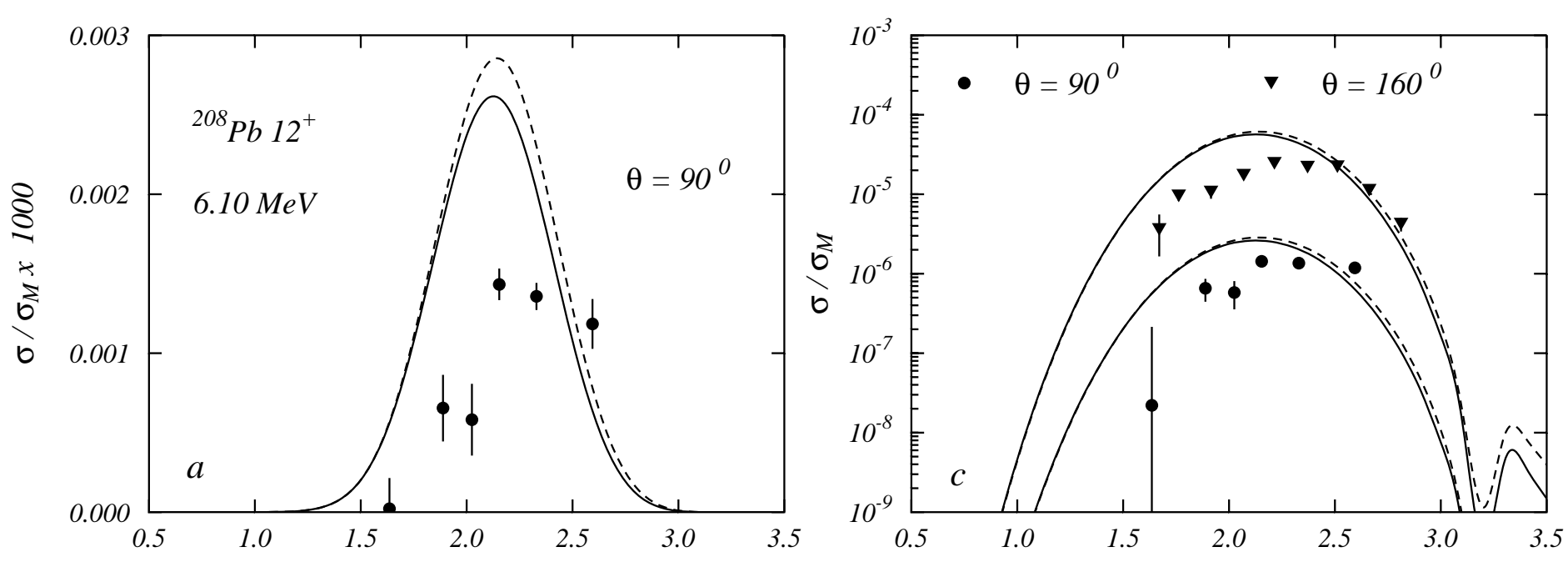

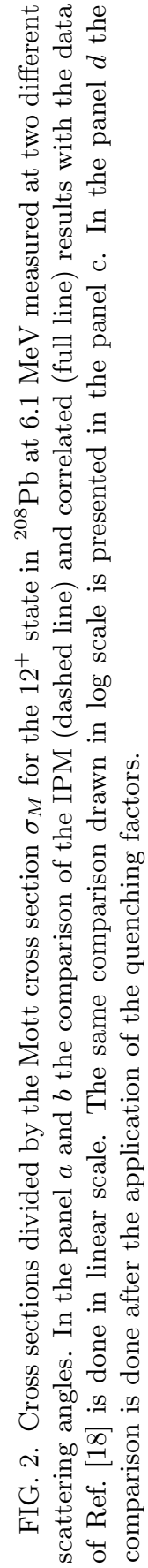
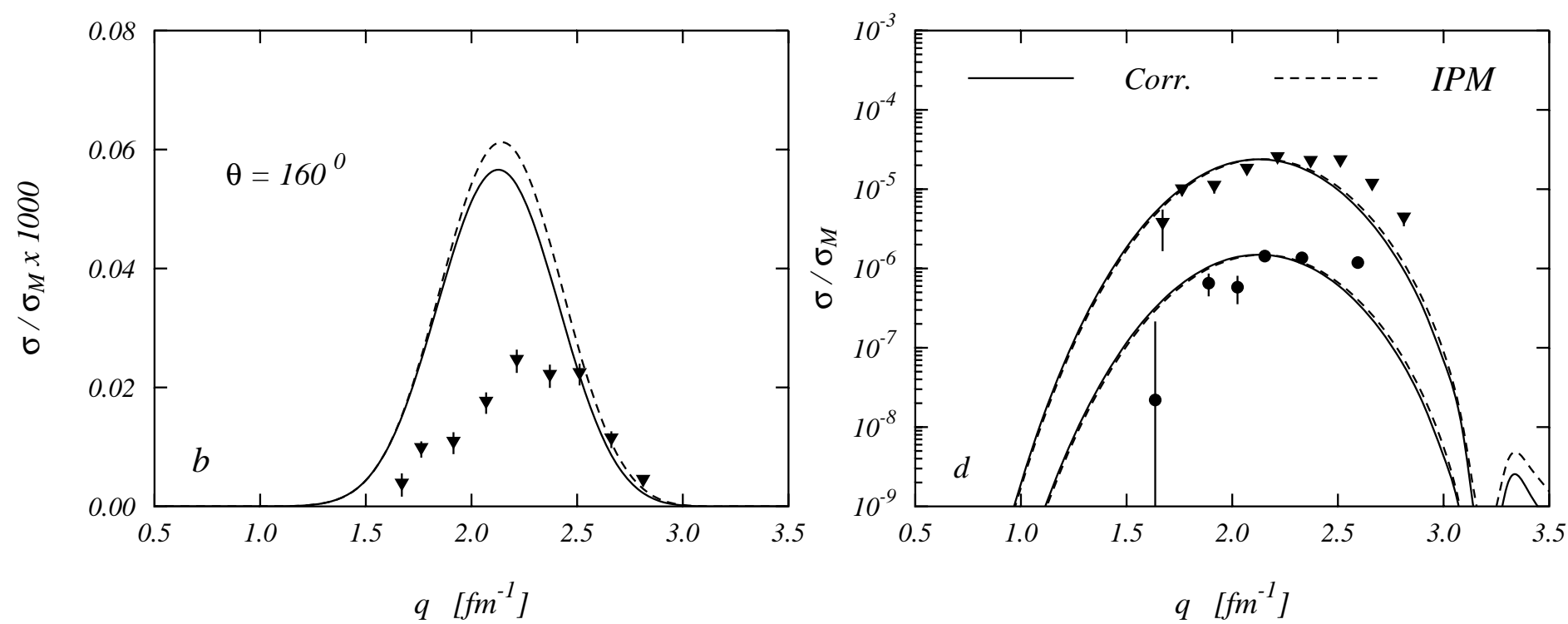

0 